\title{
Efficient control of automotive R744 heat pump using Nelder-Mead simplex method
}

\author{
GLOS, J.; VÁCLAVEK, P.
}

Proceedings of the 2017 IEEE International Conference on Industrial Technology

pp. $785-790$

ISBN: 978-1-5090-5319-3

DOl: http://dx.doi.org/10.1109/ICIT.2017.7915459

Accepted manuscript

(C2017 IEEE. Personal use of this material is permitted. Permission from IEEE must be obtained for all other uses, in any current or future media, including reprinting/republishing this material for advertising or promotional purposes, creating new collective works, for resale or redistribution to servers or lists, or reuse of any copyrighted component of this work in other works. Jan Glos, Pavel Václavek, "Efficient control of automotive R744 heat pump using Nelder-Mead simplex method", Proceedings of the 2017 IEEE International Conference on Industrial Technology, pp. 785-790, 2017. DOI: 10.1109/ICIT.2017.7915459. Final version is available at http://ieeexplore.ieee.org/ document/7915459/ 


\section{Efficient control of automotive R744 heat pump using Nelder-Mead simplex method}

\author{
Jan Glos \\ CEITEC - Central European Institute of Technology \\ Brno University of Technology \\ Brno, Czech Republic \\ Email: jan.glos@ ceitec.vutbr.cz
}

\author{
Pavel Vaclavek \\ CEITEC - Central European Institute of Technology \\ Brno University of Technology \\ Brno, Czech Republic \\ Email: pavel.vaclavek@ceitec.vutbr.cz
}

\begin{abstract}
This paper deals with coefficient of performance (COP) maximization of automotive R744 heat pump using Nelder-Mead simplex method. The COP maximum is searched using high-side pressure adjustment, what is possible and necessary during transcritical operation. Selected approach allows efficient heat pump operation, fast COP maximum localization, minimum number of needed actuator changes and resistance to heat pump parameters variation.
\end{abstract}

\section{INTRODUCTION}

Carbon dioxide $\left(\mathrm{CO}_{2}\right)$ became very interesting as a refrigerant (known as R744) since European Directive 2006/40/EC was approved. It is one of a few known refrigerants suitable for mobile air-conditioning systems (MAC) that meets requirements of this directive, especially global warming potential (GWP) lower than 150 [1]. R1234yf could be also satisfactory, but it is flammable [2]. This property can be significant disadvantage despite the fact that the risk of ignition is low [3].

R744 promising properties for both the vehicle cooling and heating were described in [4], such as some issues related to safety (high pressures), maintenance, defrosting and others were summarized. A large number of researchers investigated R744 suitability for MAC, for example [5]-[8] and especially professor Gustav Lorentzen recommended R744 as a refrigerant for automotive applications as early as in 1993 [9].

It is important to mention here $\mathrm{CO}_{2}$ basic properties. GWP is equal to 1 as R744 is being used as the reference for GWP and ozone depletion potential (ODP) is zero. The most different property (when comparing to conventional refrigerants) is very low critical point at temperature of $304.1 \mathrm{~K}$ $\left(31.1^{\circ} \mathrm{C}\right)$ and pressure of $7.38 \mathrm{MPa}$ [10]. Also $\mathrm{CO}_{2}$ has much higher volumetric heat capacity $\left(22545 \mathrm{~kJ} \mathrm{~m}^{-3}\right)$ compared to typical value (thousands) for conventional refrigerants [10]. This could be significant advantage of R744 usage especially in automotive applications, because less refrigerant is required as well as all components can be smaller and light-weight (including compressor and heat exchangers) [11].

As mentioned above, R744 has low critical point, what leads to transcritical operation at a part of operating conditions. Above critical point the pressure and the temperature are independent [4] and hence some kind of high-side pressure control is needed to achieve maximal coefficient of perfor- mance (COP). There are multiple approaches of high-side pressure control [4]. As this paper doesn't deal with refrigeration cycle design, we have chosen the basic cycle scheme with low pressure receiver. High-side pressure is controlled by adjusting the amount of refrigerant contained in high side using expansion valve.

There are multiple ways to determine the appropriate highside pressure to achieve the highest COP. The first approach employs measured temperatures (eventually pressures) and equation describing relation between temperature and pressure to compute optimal high-side pressure. As an example

$$
p_{\text {opt }}=2.759 T_{c}-9.912 \text {, }
$$

can be used as described in [12], where $p_{\text {opt }}$ is optimal highside pressure and $T_{c}$ denotes temperature at gas cooler outlet. A large number of similar and usually more complicated equations was invented (for example in [13]-[16]). As described in [17], this approach can have serious issues (up to $30 \%$ COP loss) and that is why real-time optimum searching methods are recommended.

The second approach is based on real-time (online) COP optimum searching, what can be done by extremum-seeking control (ESC), in general described in [18] and simulated for heat pump in [19]. Other perturbation-based algorithm for R744 heat pump energy efficiency optimization is described in [20]. Another solution of this problem is based on artificial neural networks [21]. Also other real-time methods based on observing changes of cooling capacity and compression work after slight variation of expansion valve were invented [22].

All of these methods of optimal high-side pressure searching have their advantages and disadvantages. Disturbance methods cause frequent changes of valve opening, other methods requires large computing resources or need some learning time to work properly. On the other hand some methods are resistant to heat pump parameter changes, can be used during transients and even some of them are very simple.

In this paper we discuss a new approach to find a COP maximum using high-side pressure variation employing NelderMead simplex method. This approach can be used in realtime, so it is able to adapt to heat pump parameters changes and it can even reflect disturbances like frozen evaporator etc. The proposed method does not require previously measured 


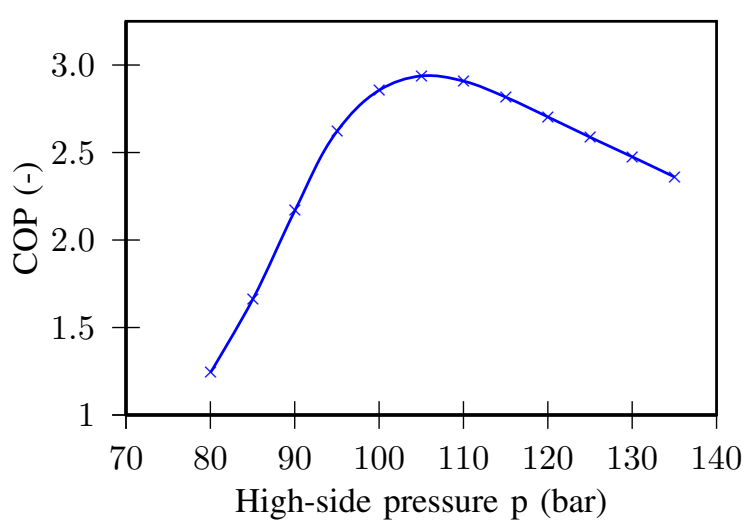

Fig. 1. COP dependence on high-side pressure under fixed conditions.

values, large computing resources nor continuous changes of valve opening. The only drawback can be impossibility to use it during fast transients (quick changes of ambient temperature and some other variables in comparison with algorithm running time) as some time (several iterations) is needed to find a COP maximum. During this time heat pump can operate with lower COP, but it should improve with algorithm progress.

\section{NELDER-MEAD SIMPLEX METHOD FOR REVERSIBLE R744 HEAT PUMP}

Nelder-Mead simplex method was chosen for COP maximum searching due to its fast convergence and easy method implementation. Usually this algorithm is used for locating a minimum of function of multiple variables. In this case we used the method to maximize a function (COP) of single variable (high-side pressure). There are slight differences for simplex method in one dimension and that is why the algorithm is described in detail in following text.

Fig. 1 illustrates COP functional dependence on highside pressure under fixed conditions (only high-side pressure changes, other variables are constant in this example). This dependence was obtained from simulation experiments as it is almost impossible to describe it using some equation due to the problem complexity. But even if successful the result might not be satisfactory (owing to possible parameter changes and disturbances as was stated in Section I). Under other conditions (ambient temperature etc.) the dependence will be little different (e.g. with some offset) as well as a COP maximum will lay at different high-side pressure, but the shape of the function will remain. It is evident from Fig. 1 that even quite small deviation from optimal pressure can cause significant COP loss (for example difference of COP for highside pressures of 105 bar and 95 bar is 0.315 ).

\section{A. R744 heat pump model}

A majority of models used in this paper was created in Dymola using Modelica language. Furthermore components from AirConditioning library were used to build a model of R744 cycle and a model of vehicle cabin. To allow external

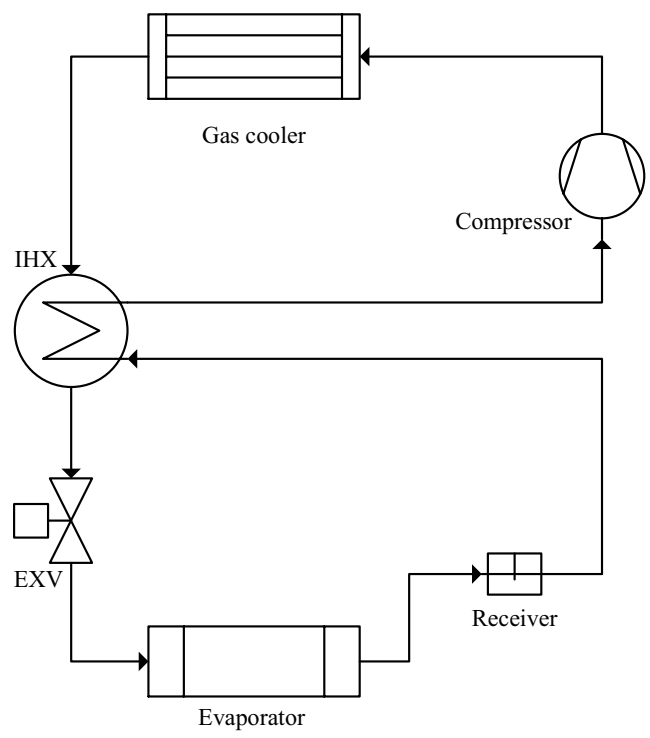

Fig. 2. R744 heat pump.

control of models and reading output values the models were complemented with blocks from standard Modelica library.

We assumed a heat pump with an electronic expansion valve $(\mathrm{EXV})$, an internal heat exchanger (IHX) and a reciprocating compressor with variable displacement. These components are supplemented with standards equipment like an evaporator, a gas cooler and a receiver. Fig. 2 shows the system configuration. Heat pump model was connected with a vehicle cabin and other HVAC equipment. We added a fan, an air valve, a duct and a solar heat flow. The air valve is designed to allow air recirculation inside the vehicle cabin. Fig. 3 illustrates the resulting circuit.

\section{B. Nelder-Mead algorithm}

A simplex method described in [23] is established to find a minimum of function of $n$ variables. It employs four operations (reflection, contraction, expansion and shrinkage) and the method is adjustable using several parameters.

Let us have a function

$$
y=f(\boldsymbol{x}),
$$

where $\boldsymbol{x}$ is a vector of independent variables and $y$ is a real function value.

A simplex is defined by $n+1$ vertices $\left(\boldsymbol{x}_{1}, \boldsymbol{x}_{2} \ldots \boldsymbol{x}_{\boldsymbol{n}}, \boldsymbol{x}_{n+1}\right)$, every having function value $\left(y_{1}, y_{2} \ldots y_{n}, y_{n+1}\right)$. The vertices are considered to be ordered ascending according to their function value from lowest $\left(x_{1}\right)$ to highest $\left(x_{n+1}\right) . \bar{x}$ is defined as a centroid of all vertices excluding $\boldsymbol{x}_{\boldsymbol{n}+\mathbf{1}}$ (vertex with highest function value). We can write

$$
\overline{\boldsymbol{x}}=\sum_{k=1}^{n} \boldsymbol{x}_{\boldsymbol{k}} / n .
$$




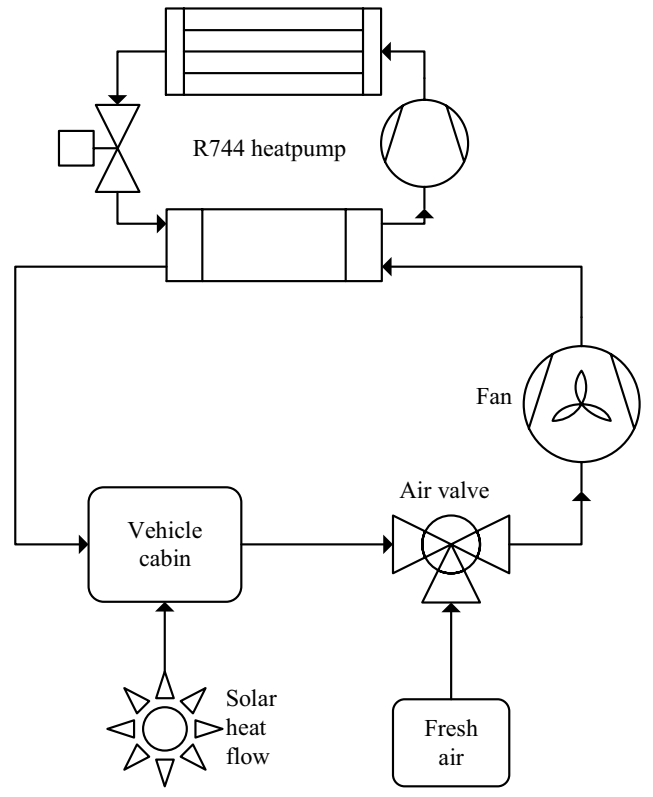

Fig. 3. R744 heat pump with vehicle cabin and HVAC equipment.

Four method coefficients are used - reflection, expansion, contraction and shrinkage coefficient $-\{\rho, \chi, \gamma, \sigma\}$ with standard values $\left\{1,2, \frac{1}{2}, \frac{1}{2}\right\}$ respectively.

1) Reflection: calculate new vertex $\boldsymbol{x}_{\boldsymbol{r}}$ using equation

$$
\boldsymbol{x}_{\boldsymbol{r}}=(1+\rho) \overline{\boldsymbol{x}}-\rho \boldsymbol{x}_{\boldsymbol{n}+\mathbf{1}},
$$

and after evaluation of $y_{r}$ select the next step based on following rules:

- if $y_{r}<y_{1}$ perform expansion

- if $y_{r} \geq y_{n+1}$ perform inside contraction

- if $y_{r} \in\left\langle y_{n}, y_{n+1}\right)$ perform outside contraction

- otherwise $\left(y_{r} \in\left\langle y_{1}, y_{n}\right)\right)$ accept $\boldsymbol{x}_{\boldsymbol{r}}$ as a new vertex instead of $\boldsymbol{x}_{\boldsymbol{n}+\mathbf{1}}$.

2) Expansion: calculate a new vertex $\boldsymbol{x}_{\boldsymbol{e}}$ using equation

$$
\boldsymbol{x}_{\boldsymbol{e}}=\chi \boldsymbol{x}_{\boldsymbol{r}}+(1-\chi) \overline{\boldsymbol{x}}=(1+\rho \chi) \overline{\boldsymbol{x}}-\rho \chi \boldsymbol{x}_{\boldsymbol{n}+\mathbf{1}},
$$

and after evaluation of $y_{e}$ make a decision

- if $y_{e}<y_{r}$, accept $\boldsymbol{x}_{\boldsymbol{e}}$

- otherwise accept $x_{r}$

instead of $\boldsymbol{x}_{\boldsymbol{n}+\mathbf{1}}$ and terminate the iteration.

3) Inside contraction: lay a new vertex $\boldsymbol{x}_{\boldsymbol{c} i}$ according to equation

$$
\boldsymbol{x}_{\boldsymbol{c i}}=\gamma \boldsymbol{x}_{\boldsymbol{n}+\mathbf{1}}+(1-\gamma) \overline{\boldsymbol{x}}=\overline{\boldsymbol{x}}-\gamma\left(\overline{\boldsymbol{x}}-\boldsymbol{x}_{\boldsymbol{n}+\mathbf{1}}\right),
$$

and then based on function value $y_{c i}$ select the next step

- if $y_{c i}<y_{n+1}$ accept new vertex $\boldsymbol{x}_{\boldsymbol{c} i}$

- otherwise perform a shrink.

4) Outside contraction: calculate a new vertex $\boldsymbol{x}_{c o}$ based on equation

$$
\boldsymbol{x}_{\boldsymbol{c o}}=\gamma \boldsymbol{x}_{\boldsymbol{r}}+(1-\gamma) \overline{\boldsymbol{x}}=\overline{\boldsymbol{x}}+\gamma \rho\left(\overline{\boldsymbol{x}}-\boldsymbol{x}_{\boldsymbol{n}+\mathbf{1}}\right),
$$

and then perform subsequent action according to function value $y_{c o}$

- if $y_{c o} \leq y_{r}$, accept new vertex $\boldsymbol{x}_{c o}$

- otherwise perform a shrink.

5) Shrinkage: replace all vertices except $\boldsymbol{x}_{1}$ (the best one) using equation

$$
\boldsymbol{x}_{\boldsymbol{i}}=\boldsymbol{x}_{\boldsymbol{1}}+\sigma\left(\boldsymbol{x}_{\boldsymbol{i}}-\boldsymbol{x}_{\mathbf{1}}\right) \quad \text { where } i=2, \ldots, n+1 .
$$

\section{Nelder-Mead algorithm for 1-dim problem}

Since our problem is one dimensional, the original NelderMead method can be considerably simplified. We found that simplex in this case degenerates to a line segment defined by two vertices. In next text the vertices are labeled $x_{h}$ (vertex with higher function value $y_{h}$ ) and $x_{l}$ (vertex with lower function value $y_{l}$ ). It is obvious that $y_{h}>y_{l}$. Regarding the centroid (3) can be adapted as

$$
\overline{\boldsymbol{x}}=\sum_{k=1}^{n} \boldsymbol{x}_{\boldsymbol{k}} / n=x_{l} .
$$

It is also clear that shrinkage coefficient and the whole step of shrinkage is not relevant for 1-dim Nelder-Mead method, because this step can not occur for strictly convex functions. That is why shrinkage is omitted in next text.

For 1-dim Nelder-Mead method we use only three coefficients for reflection, expansion and contraction - $\{\rho, \chi, \gamma\}$ with standard values $\left\{1,2, \frac{1}{2}\right\}$ respectively. All steps of 1-dim Nelder-Mead method are presented graphically in Fig. 4.

1) Reflection: calculate a new vertex $x_{r}$ using equation

$$
x_{r}=(1+\rho) x_{l}-\rho x_{h},
$$

after evaluation of $y_{r}$ select the next step based on following rules:

- if $y_{r}<y_{l}$ perform expansion

- if $y_{r} \geq y_{h}$ perform inside contraction

- if $y_{r} \in\left\langle y_{l}, y_{h}\right)$ perform outside contraction.

(For 1-dim Nelder-Mead method the vertex $y_{r}$ can be never accepted.)

2) Expansion: calculate a new vertex $x_{e}$ using equation

$$
x_{e}=\chi x_{r}+(1-\chi) x_{l}=(1+\rho \chi) x_{l}-\rho \chi x_{h},
$$

and after evaluation of $y_{e}$ make a decision

- if $y_{e}<y_{r}$, accept $x_{e}$

- otherwise accept $x_{r}$

instead of $x_{h}$ and terminate the iteration.

3) Inside contraction: lay a new vertex $x_{c i}$ according to equation

$$
x_{c i}=\gamma x_{h}+(1-\gamma) x_{l}=x_{l}-\gamma\left(x_{l}-x_{h}\right),
$$

based on function value $y_{c i}$ select the next step

- if $y_{c i}<y_{h}$ accept new vertex $x_{c i}$

- otherwise function $f$ is not strictly convex. 


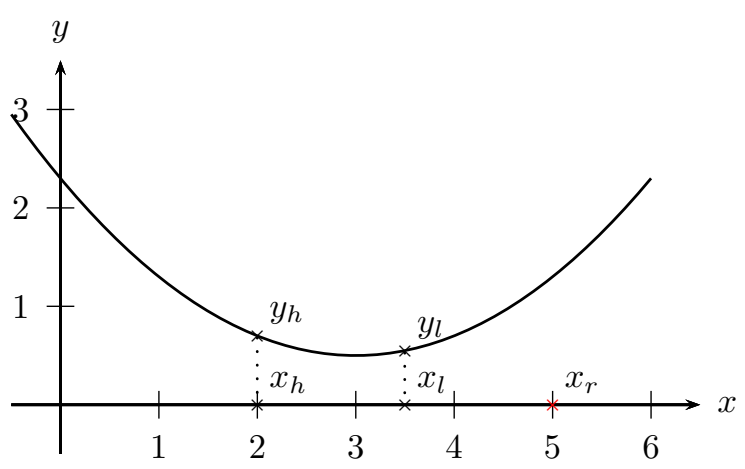

(a) Reflection

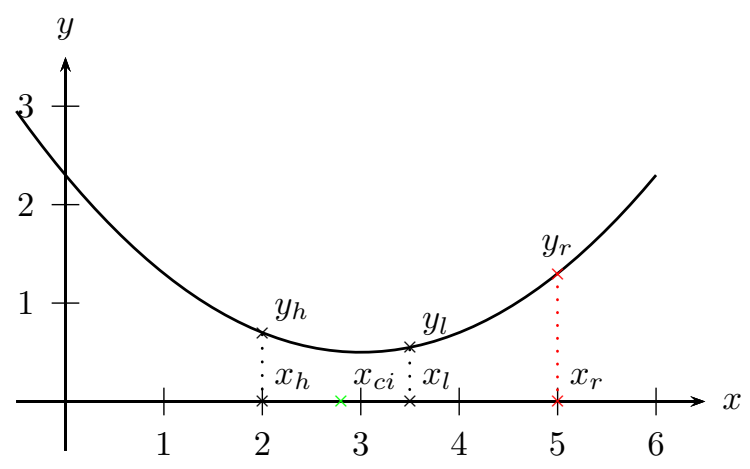

(c) Inside contraction

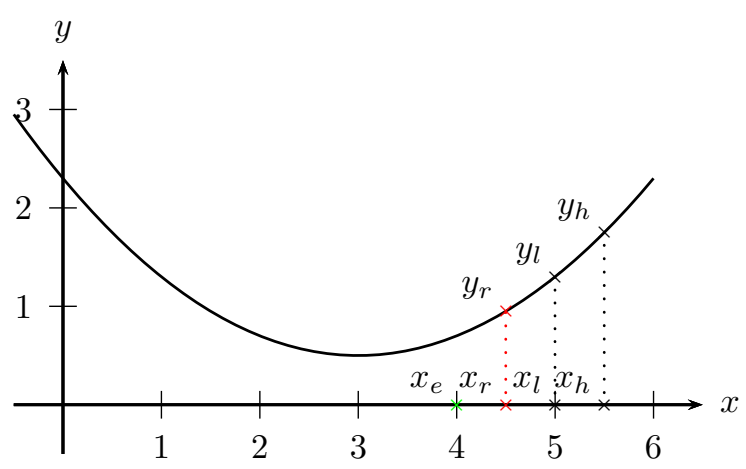

(b) Expansion

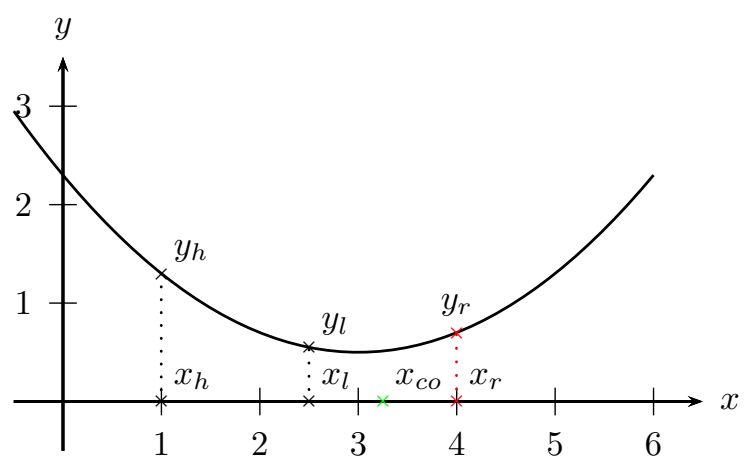

(d) Outside contraction

Fig. 4. 1-dim Nelder-Mead method steps.

4) Outside contraction: calculate a new vertex $x_{c o}$ based on equation

$$
x_{c o}=\gamma x_{r}+(1-\gamma) x_{l}=x_{l}+\gamma \rho\left(x_{l}-x_{h}\right),
$$

and then perform subsequent action according to function value $y_{c o}$

- if $y_{c o} \leq y_{r}$, accept new vertex $x_{c o}$

- otherwise function $f$ is not strictly convex.

Convergence of the simplex method in one dimension to a minimizer was proved in [24] for strictly convex functions.

\section{Control loop for $R 744$ heat pump}

Two control loops are commonly used for R744 heat pump control, first one for capacity control and the second one for high pressure control [20], [22]. We complemented them with COP feedback for Nelder-Mead method, see Fig. 5. First feedback loop controls cooling capacity and thus the temperature $(\vartheta)$ in the vehicle cabin using proportional-integral (PI) controller $P I_{\vartheta}$. As a setpoint of this controller user adjusted value of temperature $\left(\vartheta_{S P}\right)$ is used. Output $\left(c_{d}\right)$ of this controller is applied to the displacement input of the compressor. Second loop is realized using PI controller $P I_{p}$, which controls high-side pressure $(p)$. High-side pressure setpoint $\left(p_{S P}\right)$ is taken from Nelder-Mead algorithm and output of $P I_{p}$ controller is coupled to EXV as a flow factor $K_{v}$.

Transient responses of heat pump model were measured to allow PI controllers tuning. For simplicity we considered heat pump model as decoupled and linear system as the effects of coupling and nonlinearity are not very significant, so we can afford this inaccuracy. The case when the non-linear behavior of the heat-pump is considered will be subject of follow-up research. It was not possible to keep neither input at zero value, the heat pump would not work in this case. So both the inputs $\left(K_{v}\right.$ and $c_{d}$ ) were kept at some appropriate non-zero value and then we made step change of one input. This procedure was repeated for both the inputs. Thereafter transfer functions were acquired from input and output data and subsequently used for PI controllers tuning.

Nelder-Mead algorithm takes computed COP as an input value and its output is provided to controller $P I_{p}$ as a pressure setpoint. At each step of Nelder-Mead algorithm the high-side pressure setpoint is applied and COP is measured after defined time. This time delay is necessary due to settling time of highside pressure and especially COP.

\section{Simulations}

We investigated several cases of heat pump operation and for this paper we chose two of them to illustrate the COP maximum finding. 


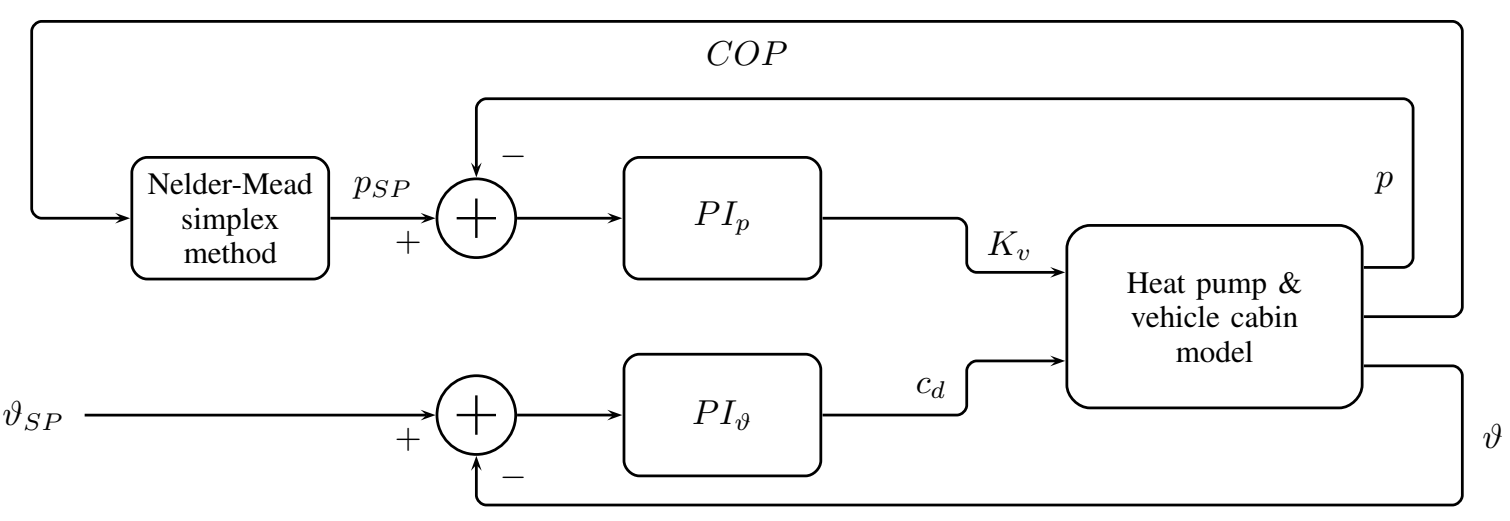

Fig. 5. R744 heat pump control loop.
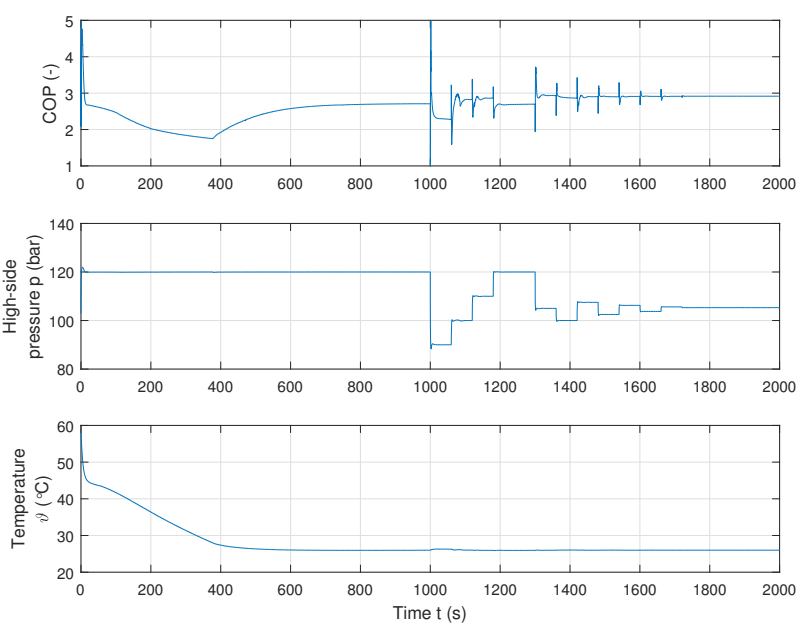

Fig. 6. Vehicle cabin cool down and COP optimization under fixed conditions and with air recirculating.

In the first case (Fig. 6) we considered heat pump operation in a hot summer day with corresponding conditions (outside air temperature $40^{\circ} \mathrm{C}$, initial temperature of cabin metal mass $45^{\circ} \mathrm{C}$ and initial cabin air temperature $58.5^{\circ} \mathrm{C}$ ). The air valve (see Fig. 3) was entirely closed, so no fresh air from outside was sucked, the air flowing to the fan was recirculated from the cabin. Result of this simulation experiment is shown in Fig. 6. At time 0 the cooling of the vehicle cabin began and after reaching steady state at time $1000 \mathrm{~s}$ the Nelder-Mead algorithm was started. The cabin temperature setpoint was constant throughout the experiment as well as the outside temperature and solar heat flow were. We chose the finishing condition of Nelder-Mead algorithm as attainment of COP difference of 0.02 for current vertices or difference of 1 bar between highside pressure setpoints. This condition was met $720 \mathrm{~s}$ after the algorithm start and resulting high-side pressure values were 105 bar and 105.5 bar, midpoint of these values was chosen as the high-side pressure setpoint. During the optimization the vehicle cabin temperature was held at the defined temperature setpoint, maximal error was approximately $0.2^{\circ} \mathrm{C}$ and there was no steady state error.
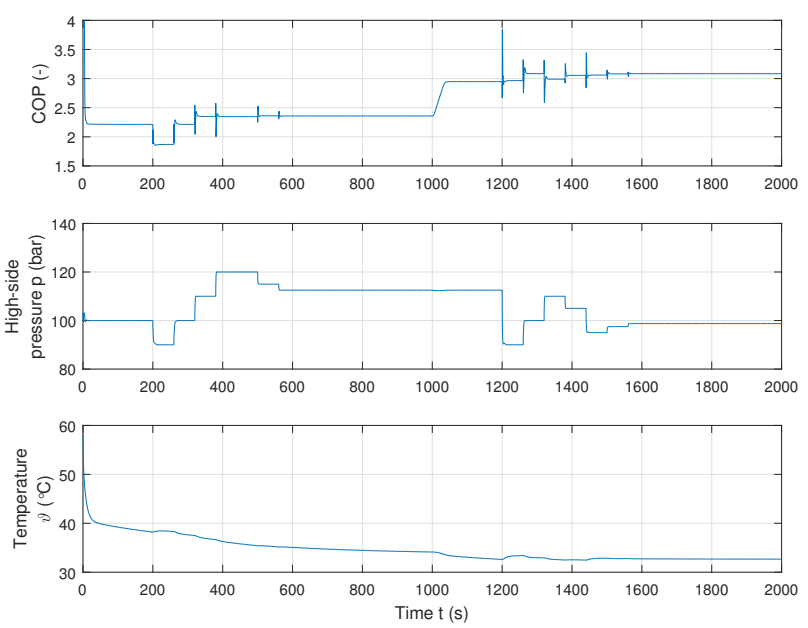

Fig. 7. Vehicle cabin cool down and COP optimization after ambient air temperature decrease (with fresh air).

In the second case (Fig. 7) the ambient conditions were the same as in the first experiment, but the air valve was completely open, only the fresh air flowed to the fan and the air from the vehicle cabin was blown out. At time $200 \mathrm{~s}$ the Nelder-Mead algorithm was started and it terminated after $360 \mathrm{~s}$. Resulting high-side pressures were 110 bar and 115 bar, average value 112.5 bar was applied as a high-side pressure setpoint. Then at time $1000 \mathrm{~s}$ the ambient temperature was decreased from $40{ }^{\circ} \mathrm{C}$ to $30^{\circ} \mathrm{C}$ employing a ramp with duration of $30 \mathrm{~s}$. After that the Nelder-Mead algorithm was restarted to find a new $\mathrm{COP}$ maximum under these new conditions. As a result high-side pressure of 98.75 bar was chosen, what is quite far from the original pressure $(112.5$ bar $)$. Using a new pressure setpoint the COP was improved by 0.14 .

\section{CONCLUSION}

In this paper we presented Nelder-Mead algorithm based method of COP maximum searching for R744 automotive heat pump operating in transcritical mode. The dynamic model consisting of the R744 heat pump and the vehicle cabin was compiled. Afterwards the resulting model was used for verification of the proposed algorithms. 
After performing the simulations we found that NelderMead simplex method can be advantageously used for COP maximum searching. It is capable to find COP maximum within few steps of high-side pressure setpoint, what can be preferable against disturbance-based methods, where the setpoint is changing continuously and so does the valve actuator. In comparison with equation based methods of COP maximum searching our method is resistant to heat pump parameters changes and other disturbances. The only disadvantage of this method is impossibility of usage during some transients, what could be solved by combining with some other COP maximizing method. It might be also possible to speed up the algorithm by reducing the time dedicated to COP evaluation in each step, what will partially remove this drawback. However it is necessary to test it on a real heat pump and determine the time required to stabilize the COP after high-side pressure change.

In future work there are multiple possibilities of improvement. We point out potential of Nelder-Mead algorithm speedup by remembering COP value of already evaluated highside pressure setpoints (see repeated evaluation of pressures 100 bar and 120 bar in Fig. 6). Another future improvement can be found regarding EXV and compressor displacement control, what leads to nonlinear multiple-input and multipleoutput (MIMO) system control (without simplifications used in this paper). Obviously the next step will be verification of these algorithms on real heat pump in laboratory environment and consequently during the real vehicle operation.

\section{ACKNOWLEDGMENT}

This research was carried out under the project H2020 653514 OSEM-EV - Optimised and Systematic Energy Management in Electric Vehicles. The research was supported by research infrastructure of CEITEC - Central European Institute of Technology.

The research results were verified in simulation using AVL CRUISE M simulation SW provided by AVL within University Partnership Program.

\section{REFERENCES}

[1] A. Mota-Babiloni, J. Navarro-Esbrí, Á. Barragán-Cervera, F. Molés, and B. Peris, "Analysis based on EU Regulation No 517/2014 of new $\mathrm{HFC} / \mathrm{HFO}$ mixtures as alternatives of high GWP refrigerants in refrigeration and HVAC systems," International Journal of Refrigeration, vol. 52, pp. 21-31, apr 2015.

[2] S. Bobbo, C. Zilio, M. Scattolini, and L. Fedele, "R1234yf as a substitute of R134a in automotive air conditioning. Solubility measurements in two commercial PAG oils," International Journal of Refrigeration, vol. 40, pp. 302-308, apr 2014.

[3] B. H. Minor, D. Herrmann, and R. Gravell, "Flammability characteristics of HFO-1234yf," Process Safety Progress, vol. 29, no. 2, pp. 150-154, jun 2010.

[4] M. H. Kim, J. Pettersen, and C. W. Bullard, "Fundamental process and system design issues in $\mathrm{CO} 2$ vapor compression systems," Progress in Energy and Combustion Science, vol. 30, no. 2, pp. 119-174, 2004.
[5] M.-Y. Lee, H.-S. Lee, and H.-P. Won, "Characteristic Evaluation on the Cooling Performance of an Electrical Air Conditioning System Using R744 for a Fuel Cell Electric Vehicle," Energies, vol. 5, no. 12, pp. 1371-1383, may 2012.

[6] T. Tamura, Y. Yakumaru, and F. Nishiwaki, "Experimental study on automotive cooling and heating air conditioning system using $\mathrm{CO} 2$ as a refrigerant," International Journal of Refrigeration, vol. 28, no. 8, pp. 1302-1307, 2005

[7] H. Lee, S. Won, Y. Ahn, and Y. Wang, "Study on the comparison of performance test result between R134a and R744 for automotive air conditioning," in ACRA 2014 - Proceedings of the 7th Asian Conference on Refrigeration and Air Conditioning. Society of Air-conditioning and Refrigerating Engineers of Korea, SAREK, 2014.

[8] F. Vetter and S. Memory, "Automotive AC/HP systems using R744 (CO 2)," in Vehicle Thermal Management Systems, VTMS 6, 2003, pp. 75-88.

[9] G. Lorentzen and J. Pettersen, "A new, efficient and environmentally benign system for car air-conditioning," International Journal of Refrigeration, vol. 16, no. 1, pp. 4-12, jan 1993.

[10] J. M. Belman-Flores, V. Pérez-García, J. F. Ituna-Yudonago, J. L. Rodríguez-Muñoz, and J. d. J. Ramírez-Minguela, "General aspects of carbon dioxide as a refrigerant," Journal of Energy in Southern Africa, vol. 25 , no. 2, pp. 96-106, 2014

[11] P. Nekså, "CO2 heat pump systems," International Journal of Refrigeration, vol. 25, no. 4, pp. 421-427, 2002.

[12] L. Yang, H. Li, S. W. Cai, L. L. Shao, and C. L. Zhang, "Minimizing COP loss from optimal high pressure correlation for transcritical $\mathrm{CO} 2$ cycle," Applied Thermal Engineering, vol. 89, pp. 656-662, 2015.

[13] F. Kauf, "Determination of the optimum high pressure for transcritical CO2-refrigeration cycles," International Journal of Thermal Sciences, vol. 38, no. 4, pp. 325-330, apr 1999.

[14] Y. Chen and J. Gu, "The optimum high pressure for $\mathrm{CO} 2$ transcritical refrigeration systems with internal heat exchangers," International Journal of Refrigeration, vol. 28, no. 8, pp. 1238-1249, 2005.

[15] S. Liao, T. Zhao, and A. Jakobsen, "A correlation of optimal heat rejection pressures in transcritical carbon dioxide cycles," Applied Thermal Engineering, vol. 20, no. 9, pp. 831-841, 2000.

[16] J. Sarkar, S. Bhattacharyya, and M. Gopal, "Optimization of a transcritical $\mathrm{CO} 2$ heat pump cycle for simultaneous cooling and heating applications," International Journal of Refrigeration, vol. 27, no. 8, pp. 830-838, 2004.

[17] L. Cecchinato, M. Corradi, and S. Minetto, "A critical approach to the determination of optimal heat rejection pressure in transcritical systems," Applied Thermal Engineering, vol. 30, no. 13, pp. 1812-1823, 2010.

[18] Kartik B. Ariyurand Miroslav Krstic, Real-time optimization by extremum-seeking control. John Wiley \& Sons, 2003.

[19] B. Hu, Y. Li, F. Cao, and Z. Xing, "Extremum seeking control of COP optimization for air-source transcritical $\mathrm{CO} 2$ heat pump water heater system," Applied Energy, vol. 147, pp. 361-372, 2015.

[20] I. Peñarrocha, R. Llopis, L. Tárrega, D. Sánchez, and R. Cabello, “A new approach to optimize the energy efficiency of $\mathrm{CO} 2$ transcritical refrigeration plants," vol. 67, no. 1-2, pp. 137-146, 2014.

[21] L. Cecchinato, M. Corradi, G. Cosi, S. Minetto, and M. Rampazzo, "A real-time algorithm for the determination of R744 systems optimal high pressure," International Journal of Refrigeration, vol. 35, no. 4, pp. 817-826, 2012.

[22] M. S. Kim, C. S. Shin, and M. S. Kim, "A study on the real time optimal control method for heat rejection pressure of a $\mathrm{CO} 2$ refrigeration system with an internal heat exchanger," International Journal of Refrigeration, vol. 48, pp. 87-99, 2014.

[23] J. Nelder, R. Mead, B. J. a. Nelder, and R. Mead, "A simplex method for function minimization," Computer Journal, vol. 7, pp. 308-313, 1964.

[24] J. Lagarias, J. Reeds, H. Wright, and P. Wright, "Convergence properties of the neldermead simplex method in low dimensions," Journal of Optimization, vol. 9, no. 1, pp. 112-117, 1998. 